\title{
GLOBAL WELLPOSEDNESS FOR THE 3D INHOMOGENEOUS INCOMPRESSIBLE NAVIER-STOKES EQUATIONS
}

\author{
WALTER CRAIG, XIANGDI HUANG, AND YUN WANG
}

\begin{abstract}
This paper addresses the three-dimensional Navier-Stokes equations for an incompressible fluid whose density is permitted to be inhomogeneous. We establish a theorem of global existence and uniqueness of strong solutions for initial data with small $\dot{H}^{\frac{1}{2}}$-norm, which also satisfies a natural compatibility condition. A key point of the theorem is that the initial density need not be strictly positive.
\end{abstract}

Keywords: inhomogeneous incompressible fluids, strong solutions, vacuum.

AMS: 35Q35, 35B65, 76N10

\section{INTRODUCTION}

In a number of important applications to fluid mechanics, such as in geophysical fluid dynamics, the Navier-Stokes equations are called upon to describe situations in which a fluid is inhomogeneous with respect to density, however it is essentially incompressible to a great extent. Such cases occur within the outer core and mantle of the earth, for example, or else within the atmosphere in a regime of flows for which dynamic effects of compressibility do not play a principle rôle. Ignoring any possible buoyancy or other body forces, flows of this character satisfy the following system of inhomogeneous incompressible Navier-Stokes equations;

$$
\left\{\begin{array}{l}
\partial_{t} \rho+\operatorname{div}(\rho u)=0 \\
\partial_{t}(\rho u)+\operatorname{div}(\rho u \otimes u)-\mu \Delta u+\nabla P=0 \\
\operatorname{div} u=0
\end{array}\right.
$$

It is a standard mathematical model to pose the initial and boundary value problem, giving the following data;

$$
\begin{gathered}
\left.(\rho, u)\right|_{t=0}=\left(\rho_{0}, u_{0}\right) \text { in } \Omega, \\
u=0 \quad \text { on } \partial \Omega, \quad u(x, \cdot) \rightarrow 0 \quad \text { as }|x| \rightarrow \infty .
\end{gathered}
$$

Here $\rho, u$ and $P$ denote the density, velocity and pressure of the fluid respectively, and $\mu$ is the positive viscosity, which is assumed to be a constant. In this paper, we consider $\Omega$ is either a smooth bounded domain in $\mathbb{R}^{3}$ or the whole space $\mathbb{R}^{3}$.

For the initial density with positive lower bound, the inhomogeneous equations (1.1) have been studied in the sixties and seventies by the Russian school, see [4,21,24] and the many references that they contain. It was proved that a unique strong solution exists locally for

The research of WC is supported in part by a Killam Research Fellowship, the Canada Research Chairs Program and NSERC through grant number 238452-11. The research of YW is supported in part by a Canada Research Chairs Postdoctoral Fellowship at McMaster University. 
arbitrary initial data. Moreover, these papers also establish global wellposedness results for small solutions in dimension $N \geq 3$, while for the two dimensional case they establish the existence of large strong solutions. More recently, there have been many subsequent contributions to this theory, obtaining global wellposedness for initial data belonging to certain scale invariant spaces, see for example [1,13, 18].

On the other hand, for initial data which permits regions of vacuum, i.e. regions where the density $\rho$ vanishes on some set, the problem becomes much more involved. Authors including DiPerna and Lions [15, 25] prove the global existence of weak solutions to (1.1) in any space dimension, see also [14,27] and the references in their work. As pointed in [12], the major difficulty for existence of strong solutions which admit regions of vacuum is the lack of an appropriate estimate for $\partial_{t} u$, since $\partial_{t} u$ only appears in the momentum equation with a possibly degenerate coefficient $\rho$. One way to avoid this is to estimate $\nabla \partial_{t} u$ instead, for which one pays the price of being required to impose a compatibility condition. In fact, Choe-Kim [12] prove the following theorem,

Theorem 1.1. Assume that the initial data $\left(\rho_{0}, u_{0}\right)$ satisfies

$$
0 \leq \rho_{0} \in L^{\frac{3}{2}} \cap H^{2}, \quad u_{0} \in D_{0}^{1,2} \cap D^{2,2},
$$

and the compatibility condition

$$
\mu \Delta u_{0}-\nabla P_{0}=\sqrt{\rho_{0}} g_{0}, \quad \operatorname{div} u_{0}=0 \quad \text { in } \Omega,
$$

for some $\left(P_{0}, g_{0}\right) \in D^{1,2} \times L^{2}$. Then there exists a time $T>0$ and a unique strong solution $(\rho, u, P)$ to the initial boundary value problem (1.1)-(1.2) satisfying

$$
\begin{gathered}
\rho \in L^{\infty}\left(0, T ; L^{\frac{3}{2}} \cap H^{2}\right), \quad u \in C\left([0, T] ; D_{0, \sigma}^{1,2} \cap D^{2,2}\right) \cap L^{2}\left(0, T ; D^{3,2}\right), \\
\partial_{t} u \in L^{2}\left(0, T ; D_{0, \sigma}^{1,2}\right), \quad \text { and } \sqrt{\rho} \partial_{t} u \in L^{\infty}\left(0, T ; L^{2}\right) .
\end{gathered}
$$

It is also interesting to study the regularity criterion for such strong solutions, see [11,22] and its references. In particular, Kim 22] proved that if $T^{*}$ is the blowup time of a local strong solution, then necessarily

$$
\int_{0}^{T^{*}}\|u(s)\|_{L^{q, \infty}}^{p} d s=\infty, \quad \text { for any }(p, q), \quad \frac{2}{p}+\frac{3}{q}=1,3<q \leq \infty,
$$

where $L^{q, \infty}$ is the usual weak Lebesgue space. This is effectively a criterion of regularity, and is similar to the Serrin criterion [26] for a priori regularity condition for the homogeneous Navier-Stokes equations.

However, like the usual homogeneous Navier-Stokes equations, the question of global existence of strong solutions remains open for large initial data. A partial result due to Kim [22] asserts that if $\left\|u_{0}\right\|_{D^{1,2}}$ is small enough, then a strong solution exists globally in time and is unique. In this paper, we generalize this result to the case of data within a class of critical Sobolev spaces.

There is a scaling invariance of the system (1.1), namely if $(\rho, u, P)$ is a solution associated with the initial data $\left(\rho_{0}, u_{0}\right)$, then

$$
\left(\rho_{\lambda}, u_{\lambda}, P_{\lambda}\right)=\left(\rho\left(\lambda^{2} t, \lambda x\right), \lambda u\left(\lambda^{2} t, \lambda x\right), \lambda^{2} P\left(\lambda^{2} t, \lambda x\right)\right)
$$


is a solution to (1.1)-(1.2) associated with the initial data $\left(\rho_{0}(\lambda x), \lambda u_{0}(\lambda x)\right)$. A scale invariant function space $H^{*}$ is one for which $\left\|\left(\rho_{\lambda}, u_{\lambda}\right)\right\|_{H^{*}}=\left\|\left(\rho_{1}, u_{1}\right)\right\|_{H^{*}}$. For example, scaling invariance implies that

$$
\|u\|_{\dot{H}^{\frac{1}{2}}}=\left\|u_{\lambda}\right\|_{\dot{H}^{\frac{1}{2}}}
$$

which is our principle example. Our main result in this paper is that if $\left\|u_{0}\right\|_{\dot{H}^{\frac{1}{2}}}$ is small enough, then a strong solution exists globally in time. More precisely,

Theorem 1.2. Assume the conditions in Theorem 1.1. Then there exists a small constant $\varepsilon$ depending on $\bar{\rho}=\left\|\rho_{0}\right\|_{L^{\infty}}, \mu$ and the domain $\Omega$, such that if

$$
\left\|u_{0}\right\|_{\dot{H}^{\frac{1}{2}}} \leq \varepsilon,
$$

then the unique local strong solution constructed in Theorem 1.1 exists globally in time. Moreover, this global strong solution satisfies the following decay properties:

$$
\|\nabla u\|_{L^{2}} \leq M t^{-\frac{1}{2}}
$$

where $M$ depends on $\mu, \Omega, \bar{\rho}$, and $\int \rho_{0}\left|u_{0}\right|^{2} d x$.

The global result above relies on the smallness of the scale invariant norm of the initial data. In terms of the well known result of Fujita \& Kato [16] for the homogeneous NavierStokes equations, our result can be considered as its generalization to the inhomogeneous case. The homogeneous Navier-Stokes equations have been a much more active topic of inquiry in this direction, see [9, 10, 23] and their references. The key ideas leading to these results come from a variety of refined estimates of Stokes semigroup combined with the contraction principle for a Picard iteration scheme. However, in the present work one can not directly apply the same methods, due to the presence of the additional factor $\rho$ before the term $\partial_{t} u$. Instead we pursue a different strategy of proof, based on the method of energy estimates, and using the parabolic property of the system of equations. The main idea goes as follows: we first assume that the scale-invariant quantity $\|\nabla u\|_{L_{t}^{4} L_{x}^{2}}$ is less than an a priori constant bound 2; then using this assumption we then prove, under the smallness assumption on the initial data, that in fact the quantity is less than 1 . Since $\|\nabla u\|_{L_{t}^{4} L_{x}^{2}}$ is initially 0 , which is less than 1, then it remains less than 1. On the other hand, the boundedness of $\|\nabla u\|_{L_{t}^{4} L_{x}^{2}}$ implies the local solution can be extended, according to some a priori estimates.

The proof in this paper is inspired by a similar result for 3D compressible Navier-Stokes equations due to Huang, Li \& Xin [19], in which they proved the global wellposedness of classical solutions with small initial $H^{\frac{1}{2}+\varepsilon}$-norm.

Remarks: Compared to the global existence results in [1,13, 18], for which the initial density is required to be a small perturbation of a constant, our result does not require an assumption of smallness on the density variations, and in fact it allows for the presence of regions of vacuum.

In the case of periodic boundary conditions on a torus $\mathbb{T}^{d}$, the same result can be proved if the intial data has zero momentum, following the strategy as for the case of a bounded domain. Indeed, the same conclusion holds for classical solutions if the initial data are regular enough and satisfy some higher-order compatibility conditions. The proof of this statement is almost the same except that some additional high-order estimates are required. These 
estimates have been given in [22]. In the case in which $\Omega$ is a smooth bounded domain in $\mathbb{R}^{2}$, it is already known that global strong solutions exist without a smallness assumption; this was proved first for the case without vacuum [4] and subsequently for the case with vacuum [20].

Our result holds in particular in the case of a constant density $\rho=1$. It therefore gives a new proof of the well known Fujita-Kato theorem [16] , which uses only energy estimate methods in the argument.

Theorem 1.3. Assume $u_{0} \in H^{1}\left(\mathbb{R}^{3}\right)$ and $\operatorname{div} u_{0}=0$. There exists a positive constant $\varepsilon$, such that if $\left\|u_{0}\right\|_{\dot{H}^{\frac{1}{2}}} \leq \varepsilon$, then there is a global strong solution to the homogeneous Navier-Stokes equations with the property that

$$
u \in C\left([0, \infty) ; H^{1}\right) \cap L_{l o c}^{2}\left(0, \infty ; H^{2}\right) .
$$

This article is organized as follows: section 2 sets the notation, and contains several definitions and basic lemmas. In section 3 we give the proof for the case $\Omega=\mathbb{R}^{3}$, while the proof for the case of a bounded domain is presented in section 4 .

\section{Notation And interpolation LEMmas}

The homogeneous and inhomogeneous Sobolev spaces are defined in the standard way. For $1 \leq r \leq \infty$ and $k \in \mathbb{N}$,

$$
\begin{aligned}
& L^{r}=L^{r}(\Omega), \\
& D^{k, r}=\left\{u \in L_{l o c}^{1}:\left\|\nabla^{k} u\right\|_{L^{r}}<\infty\right\}, \quad\|u\|_{D^{k, r}=\left\|\nabla^{k} u\right\|_{L^{r}}}, \\
& W^{k, r}=L^{r} \cap D^{k, r}, \quad H^{k}=W^{k, 2}, \quad D_{0}^{k, r}=\overline{C_{0}^{\infty}} \text { closure in the norm of } D^{k, r} .
\end{aligned}
$$

In particular when $\Omega$ is a bounded domain then $D_{0}^{1,2}=H_{0}^{1}$. Set

$$
C_{0, \sigma}^{\infty}=\left\{u \in C_{0}^{\infty}: \operatorname{div} u=0\right\},
$$

and set $D_{\sigma}^{1,2}=\overline{C_{0, \sigma}^{\infty}}$, with the closure taken in the norm of $D^{1,2}$. The fractional-order homogeneous Sobolev space $\dot{H}^{s}\left(\mathbb{R}^{3}\right)$ are defined as the space of tempered distributions $u$ over $\mathbb{R}^{3}$ for which the Fourier transform $\mathcal{F} u$ belongs to $L_{l o c}^{1}\left(\mathbb{R}^{3}\right)$ and which satisfy

$$
\|u\|_{\dot{H}^{s}\left(\mathbb{R}^{3}\right)}^{2}:=\int_{\mathbb{R}^{3}}|\xi|^{2 s}|\mathcal{F} u(\xi)|^{2} d \xi<\infty .
$$

And $\dot{H}^{s}(\Omega)$ is the restriction of $\dot{H}^{s}\left(\mathbb{R}^{3}\right)$ to the domain $\Omega$. We refer to $\dot{H}^{s}(\Omega)$ by $\dot{H}^{s}$, independent of whether $\Omega$ is the whole space or not.

Some well known interpolation results which relate Lorentz spaces and the classical Sobolev spaces are presented, which can be found in [8,28,29]. For every $0<\theta<1,1 \leq q \leq \infty$, and normed spaces $X_{0}, X_{1}$, we use the notation $\left[X_{0}, X_{1}\right]_{\theta, q}$ to denote the real interpolation space between $X_{0}$ and $X_{1}$.

Lemma 2.1. Let $s, s_{0}, s_{1} \in \mathbb{R}$. If $s=(1-\theta) s_{0}+\theta s_{1}$ with some $0<\theta<1$, then

$$
\left[\dot{H}^{s_{0}}, \dot{H}^{s_{1}}\right]_{\theta, 2}=\dot{H}^{s} \text {. }
$$


And the following inclusion relation holds, if $1 \leq q_{1} \leq q_{2} \leq \infty$, then

$$
\left[\dot{H}^{s_{0}}, \dot{H}^{s_{1}}\right]_{\theta, q_{1}} \subseteq\left[\dot{H}^{s_{0}}, \dot{H}^{s_{1}}\right]_{\theta, q_{2}} .
$$

Lemma 2.2. Let $0<\theta<1,1<p_{0}, p_{1}, p<\infty, 1 \leq q_{0}, q_{1}, q \leq \infty$, and $\frac{1}{p}=\frac{1-\theta}{p_{0}}+\frac{\theta}{p_{1}}$, then

$$
\left[L^{p_{0}, q_{0}}, L^{p_{1}, q_{1}}\right]_{\theta, q}=L^{p, q} .
$$

This identification of the interpolation spaces (2.8) is also valid if $L^{p_{1}, q_{1}}$ is replaced by by $L^{\infty}\left(L^{p_{0}, q_{0}}, L^{p_{1}, q_{1}}\right.$ and $L^{p, q}$ are Lorentz spaces, whose definition can be found in [8]). When $q=p, L^{p, p}$ is the Sobolev $L^{p}$ space.

The above interpolation lemmas clearly generalize to vector-valued Lorentz spaces. For example,

Lemma 2.3. Let $0<\theta<1,1<p_{0}, p_{1}<\infty, 1 \leq q_{0}, q_{1}, q \leq \infty$, and $\frac{1}{p}=\frac{1-\theta}{p_{0}}+\frac{\theta}{p_{1}}$. Then

$$
\left[L^{p_{0}, q_{0}}\left(0, T ; L^{2}\right), L^{p_{1}, q_{1}}\left(0, T ; L^{2}\right)\right]_{\theta, q}=L^{p, q}\left(0, T ; L^{2}\right) .
$$

The statement (2.9) is also valid after replacing $L^{p_{1}, q_{1}}\left(0, T ; L^{2}\right)$ by $L^{\infty}\left(0, T ; L^{2}\right)$.

Bounds for linear operators on interpolation spaces are the concern of the next lemma.

Lemma 2.4. Suppose that $E_{0}, E_{1}, F_{0}, F_{1}$ are Banach spaces and $0<\theta<1,1 \leq q \leq \infty$, and that $A$ is a linear operator from $E_{0}+E_{1}$ into $F_{0}+F_{1}$. If $A$ maps $E_{0}$ into $F_{0}$, with $\|A w\|_{F_{0}} \leq L_{0}\|w\|_{E_{0}}$ for all $w \in E_{0}$, and maps $E_{1}$ into $F_{1}$, with $\|A w\|_{F_{1}} \leq L_{1}\|w\|_{E_{1}}$ for all $w \in E_{1}$. Then $A$ is a continous linear operator from $\left[E_{0}, E_{1}\right]_{\theta, q}$ into $\left[F_{0}, F_{1}\right]_{\theta, q}$ and one has

$$
\|A w\|_{\left[F_{0}, F_{1}\right]_{\theta, q}} \leq L_{0}^{1-\theta} L_{1}^{\theta}\|w\|_{\left[E_{0}, E_{1}\right]_{\theta, q}} .
$$

In this paper, the constants $C, C_{1}, C_{2}, C_{3}, C_{4}, C_{5}, \cdots$ may depend upon $\mu, \bar{\rho}, \Omega$ and as conventional in analysis, they may change from line to line.

\section{Proof of Theorem 1.2 for $\Omega=\mathbb{R}^{3}$}

In this section, we focus on the case $\Omega=\mathbb{R}^{3}$.

3.1. A Priori Estimates. This subsection establishes several a priori estimates for strong solutions to the Cauchy problem, which will play a key role in extending local strong solutions to global ones.

Given a strong solution $(\rho, u, P)$ on $\mathbb{R}^{3} \times[0, T]$, define

$$
A(t)=\|\nabla u\|_{L^{4}\left(0, t ; L^{2}\right)}, \quad 0 \leq t \leq T .
$$

Theorem 3.1. Under the assumptions of Theorem 1.1, there exists a positive constant $\varepsilon$ depending only on $\mu$ and $\bar{\rho}$, such that if $\left\|u_{0}\right\|_{\dot{H}^{\frac{1}{2}}} \leq \varepsilon$, and $(\rho, u, P)$ is a strong solution to (1.1) -(1.2), satisfying

$$
A(T) \leq 2,
$$

then it in fact holds that

$$
A(T) \leq 1
$$

The remainder of this subsection consists in proving this key result. 
Lemma 3.2. Let $(\rho, u, P)$ be a strong solution of (1.1) -(1.2). Then for all $0 \leq t \leq T$,

$$
\|\rho(t)\|_{L^{\infty}}=\left\|\rho_{0}\right\|_{L^{\infty}}=\bar{\rho} .
$$

Proof. The mass equation is in fact a transport equation, owing to the fact that $\operatorname{div}(u)=0$, from which (3.12) follows.

Lemma 3.3. Let $(\rho, u, P)$ be a strong solution of (1.1)-(1.2). Suppose that

$$
A(T) \leq 2,
$$

then there exists some constant $C_{1}$ depending on $\mu, \bar{\rho}$, such that

$$
\|\nabla u\|_{L^{4}\left(0, T ; L^{2}\right)} \leq C_{1}\left\|u_{0}\right\|_{\dot{H}^{\frac{1}{2}}}
$$

and it holds that

$$
\sup _{t \in[0, T]} t|| \nabla u(t) \|_{L^{2}}^{2} \leq C \int \rho_{0}\left|u_{0}\right|^{2} d x
$$

Note that $C_{1}$ does not depend on $T$.

Proof. First, let's consider the following linear Cauchy problem for $(w, \tilde{P})$,

$$
\left\{\begin{array}{l}
\rho \partial_{t} w-\mu \Delta w+(\rho u \cdot \nabla) w+\nabla \tilde{P}=0 \\
\operatorname{div} w=0 \\
w(x, 0)=w_{0}(x)
\end{array}\right.
$$

Suppose that $w_{0}$ satisfies the conditions assumed for $u_{0}$ as prescribed in Theorem 1.1, then the existence and uniqueness of strong solution to (3.15) has been proved in [12. Straightforward energy estimates tell that

$$
\frac{1}{2} \int \rho|w(T)|^{2} d x+\mu \int_{0}^{T}\|\nabla w\|_{L^{2}}^{2} d t \leq \frac{1}{2} \int \rho_{0}\left|w_{0}\right|^{2} d x \leq C(\bar{\rho})\left\|w_{0}\right\|_{L^{2}}^{2} .
$$

Multiplying (3.15) 1 by $\partial_{t} w$ and integrating over $\mathbb{R}^{3}$, one gets by Sobolev embedding that

$$
\begin{array}{rl}
\int \rho\left|\partial_{t} w\right|^{2} & d x+\frac{\mu}{2} \frac{d}{d t} \int|\nabla w|^{2} d x=-\int(\rho u \cdot \nabla) w \cdot w_{t} d x \\
& \leq C(\bar{\rho})\|u\|_{L^{6}}\|\nabla w\|_{L^{3}}\left\|\sqrt{\rho} \partial_{t} w\right\|_{L^{2}} \\
& \leq \frac{1}{4} \int \rho\left|\partial_{t} w\right|^{2} d x+C\|\nabla u\|_{L^{2}}^{2}\|\nabla w\|_{L^{2}}\|\nabla w\|_{L^{6}} .
\end{array}
$$

Notice that the momentum equation can be written as

$$
-\mu \Delta w+\nabla \tilde{P}=-\rho \partial_{t} w-(\rho u \cdot \nabla) w,
$$

where the left handside is viewed as the Helmholtz-Weyl decomposition of the right one. From this equation,

$$
\mu\|\Delta w\|_{L^{2}} \leq\left\|\rho \partial_{t} w\right\|_{L^{2}}+\|(\rho u \cdot \nabla) w\|_{L^{2}} .
$$


It follows from Calderón-Zygmund inequality and the Sobolev embedding theorem that

$$
\begin{aligned}
& \|\nabla w\|_{L^{6}} \leq C\left\|\nabla^{2} w\right\|_{L^{2}} \\
\leq & C\left\|\rho \partial_{t} w\right\|_{L^{2}}+C\|(\rho u \cdot \nabla) w\|_{L^{2}} \\
\leq & C\left\|\rho \partial_{t} w\right\|_{L^{2}}+C\|\nabla u\|_{L^{2}}\|\nabla w\|_{L^{2}}^{\frac{1}{2}}\|\nabla w\|_{L^{6}}^{\frac{1}{2}},
\end{aligned}
$$

which, together with Young's inequality, implies that

$$
\|\nabla w\|_{L^{6}} \leq C\left\|\nabla^{2} w\right\|_{L^{2}} \leq C\left\|\rho \partial_{t} w\right\|_{L^{2}}+C\|\nabla u\|_{L^{2}}^{2}\|\nabla w\|_{L^{2}} .
$$

Inserting (3.19) into (3.17), we get

$$
\frac{1}{2} \int \rho\left|\partial_{t} w\right|^{2} d x+\frac{\mu}{2} \frac{d}{d t} \int|\nabla w|^{2} d x \leq C\|\nabla u\|_{L^{2}}^{4}\|\nabla w\|_{L^{2}}^{2} .
$$

By Gronwall's inequality and the assumption $A(T) \leq 2$,

$$
\int_{0}^{t} \int \rho\left|\partial_{t} w(s)\right|^{2} d x d s+\|\nabla w(t)\|_{L^{2}}^{2} \leq C e^{C}\left\|\nabla w_{0}\right\|_{L^{2}}^{2} .
$$

For fixed $(\rho, u)$, the map from $w_{0}$ to $\nabla w(t)$ is linear. Furthermore, by Lemmas 2.1 and 2.3, $L^{4}\left(0, T ; L^{2}\right)=\left[L^{2}\left(0, T ; L^{2}\right), L^{\infty}\left(0, T ; L^{2}\right)\right]_{\frac{1}{2}, 4}$ and $\dot{H}^{\frac{1}{2}} \subseteq\left[L^{2}, \dot{H}^{1}\right]_{\frac{1}{2}, 4}$, then one gets, upon combining the estimate (3.16) and (3.21), and using Lemma 2.4, that

$$
\|\nabla w\|_{L^{4}\left(0, T ; L^{2}\right)} \leq C_{1}\left\|w_{0}\right\|_{\dot{H}^{\frac{1}{2}}} .
$$

Notice that $C_{1}$ does not depend on $T$, since one can scale $[0, T]$ to $[0,1]$. Consequently,

$$
\|\nabla u\|_{L^{4}\left(0, T ; L^{2}\right)} \leq C_{1}\left\|u_{0}\right\|_{\dot{H}^{\frac{1}{2}}} .
$$

Regarding the decay of $\|\nabla u\|_{L^{2}}$, upon multiplying (3.20) by $t$, one gets that

$$
t \int \rho\left|\partial_{t} u\right|^{2} d x+\frac{d}{d t} \int t|\nabla u|^{2} d x \leq \int|\nabla u|^{2} d x+C t\|\nabla u\|_{L^{2}}^{4}\|\nabla u\|_{L^{2}}^{2} .
$$

If $A(T) \leq 2$, then by Gronwall's inequality,

$$
\begin{aligned}
& \int_{0}^{T} t\left\|\sqrt{\rho} \partial_{t} u\right\|_{L^{2}}^{2} d t+\sup _{t \in[0, T]} t\|\nabla u(t)\|_{L^{2}}^{2} \\
& \leq C \int_{0}^{T}\|\nabla u\|_{L^{2}}^{2} d t \leq C \int \rho_{0}\left|u_{0}\right|^{2} d x,
\end{aligned}
$$

where the basic energy inequality for $u$ is utilized.

Proof of Theorem 3.1. The conclusion of Theorem 3.1] will follow if we let $\varepsilon \leq 1 / C_{1}$, which is given in Lemma 3.3.

Remark 3.1. According to the proof of Lemma 3.3, the $\dot{H}^{\frac{1}{2}}$-norm of $u_{0}$ can be replaced by $\dot{B}_{2,4}^{\frac{1}{2}}$-norm, which will make the main result more refined. 
From this point on, we will consider only the small data problem, assuming that the initial data satisfies the condition $\left\|u_{0}\right\|_{\dot{H}^{\frac{1}{2}}} \leq \varepsilon$, as in Theorem 3.1. The notation $\bar{C}$ is used to denote a positive constant, which may depend on $T$ and the initial data, and it may change from line to line. The computation is standard, with respect to that in [22], but we sketch it here for completeness. From the proof of Lemma 3.3, we know that

$$
\int_{0}^{T}\left\|\sqrt{\rho} \partial_{t} u\right\|_{L^{2}}^{2} d t+\mu\|\nabla u\|_{L^{\infty}\left(0, T ; L^{2}\right)}^{2} \leq C\left\|\nabla u_{0}\right\|_{L^{2}}^{2} \leq \bar{C} .
$$

Lemma 3.4 (Estimates for $\left\|\sqrt{\rho} \partial_{t} u\right\|_{L^{2}}$ and $\left.\|\nabla u\|_{H^{1}}\right)$. Under the assumption of Theorem 3.1, we have

$$
\sup _{t \in[0, T]}\left[\left\|\sqrt{\rho} \partial_{t} u\right\|_{L^{2}}^{2}+\|\nabla u\|_{H^{1}}^{2}\right]+\int_{0}^{T}\left\|\nabla \partial_{t} u\right\|_{L^{2}}^{2} d t \leq \bar{C}
$$

Proof. Differentiating the momentum equation with respect to $t$, multiplying by $\partial_{t} u$, and then integrating over $\mathbb{R}^{3}$, one can obtain that

$$
\begin{aligned}
& \frac{1}{2} \frac{d}{d t} \int \rho\left|\partial_{t} u\right|^{2} d x+\mu \int\left|\nabla \partial_{t} u\right|^{2} d x \\
= & -2 \int \rho u \cdot \nabla \partial_{t} u \cdot \partial_{t} u d x-\int \rho u \cdot \nabla\left(u \cdot \nabla u \cdot \partial_{t} u\right) d x-\int\left(\rho \partial_{t} u \cdot \nabla\right) u \cdot \partial_{t} u d x .
\end{aligned}
$$

It follows from Sobolev embedding theorem and Gagliardo-Nirenberg inequality that

$$
\begin{aligned}
& -2 \int \rho u \cdot \nabla \partial_{t} u \cdot \partial_{t} u d x \\
& \leq C\|\nabla u\|_{L^{2}}\left\|\nabla \partial_{t} u\right\|_{L^{2}}^{\frac{3}{2}}\left\|\sqrt{\rho} \partial_{t} u\right\|_{L^{2}}^{\frac{1}{2}} \\
& \leq \frac{\mu}{8}\left\|\nabla \partial_{t} u\right\|_{L^{2}}^{2}+C\|\nabla u\|_{L^{2}}^{4}\left\|\sqrt{\rho} \partial_{t} u\right\|_{L^{2}}^{2} .
\end{aligned}
$$

By Sobolev embedding and the estimate (3.19), the second term can be estimated,

$$
\begin{aligned}
& -\int \rho u \cdot \nabla\left(u \cdot \nabla u \cdot \partial_{t} u\right) d x \\
& \quad \leq \int \rho\left|u \left\|\left.\nabla u\right|^{2}\left|\partial_{t} u\right| d x+\int \rho|u|^{2}\left|\nabla ^ { 2 } u \left\|\left.\partial_{t} u\left|d x+\int \rho\right| u\right|^{2}\left|\nabla u \| \nabla \partial_{t} u\right| d x\right.\right.\right.\right. \\
& \leq C\|u\|_{L^{6}}\|\nabla u\|_{L^{2}}\|\nabla u\|_{L^{6}}\left\|\partial_{t} u\right\|_{L^{6}}+C\|u\|_{L^{6}}^{2}\left\|\nabla^{2} u\right\|_{L^{2}}\left\|\partial_{t} u\right\|_{L^{6}} \\
& \quad+C\|u\|_{L^{6}}^{2}\|\nabla u\|_{L^{6}}\left\|\nabla \partial_{t} u\right\|_{L^{2}} \\
& \quad \leq C\|\nabla u\|_{L^{2}}^{4}\left\|\nabla^{2} u\right\|_{L^{2}}^{2}+\frac{\mu}{8}\left\|\nabla \partial_{t} u\right\|_{L^{2}}^{2} \\
& \quad \leq C\|\nabla u\|_{L^{2}}^{4}\left\|\rho \partial_{t} u\right\|_{L^{2}}^{2}+C\|\nabla u\|_{L^{2}}^{10}+\frac{\mu}{8}\left\|\nabla \partial_{t} u\right\|_{L^{2}}^{2} .
\end{aligned}
$$


For the third term on the right handside of (3.26), utilizing Gagliardo-Nirenberg inequality and (3.19),

$$
\begin{aligned}
& -\int\left(\rho \partial_{t} u \cdot \nabla\right) u \cdot \partial_{t} u d x \leq C\left\|\sqrt{\rho} \partial_{t} u\right\|_{L^{2}}\|\nabla u\|_{L^{6}}\left\|\sqrt{\rho} \partial_{t} u\right\|_{L^{3}} \\
& \quad \leq C\left\|\sqrt{\rho} \partial_{t} u\right\|_{L^{2}}^{\frac{3}{2}}\left\|\nabla \partial_{t} u\right\|_{L^{2}}^{\frac{1}{2}}\left(\left\|\rho \partial_{t} u\right\|_{L^{2}}+\|\nabla u\|_{L^{2}}^{3}\right) \\
& \quad \leq C\left\|\sqrt{\rho} \partial_{t} u\right\|_{L^{2}}^{\frac{10}{3}}+C\left\|\sqrt{\rho} \partial_{t} u\right\|_{L^{2}}^{2}\|\nabla u\|_{L^{2}}^{4}+\frac{\mu}{8}\left\|\nabla \partial_{t} u\right\|_{L^{2}}^{2} .
\end{aligned}
$$

Collecting all the estimates (3.27)-(3.29), one gets that

$$
\begin{aligned}
& \frac{1}{2} \frac{d}{d t} \int \rho\left|\partial_{t} u\right|^{2} d x+\frac{\mu}{2} \int\left|\nabla \partial_{t} u\right|^{2} d x \\
& \quad \leq C\|\nabla u\|_{L^{2}}^{4}\left\|\sqrt{\rho} \partial_{t} u\right\|_{L^{2}}^{2}+C\left\|\sqrt{\rho} \partial_{t} u\right\|_{L^{2}}^{\frac{4}{3}}\left\|\sqrt{\rho} \partial_{t} u\right\|_{L^{2}}^{2}+C\|\nabla u\|_{L^{2}}^{10} .
\end{aligned}
$$

Utilizing Gronwall's inequality and the estimate (3.24), we obtain that

$$
\sup _{t \in[0, T]}\left\|\sqrt{\rho} \partial_{t} u\right\|_{L^{2}}^{2}+\int_{0}^{T}\left\|\nabla \partial_{t} u\right\|_{L^{2}}^{2} d t \leq \bar{C} .
$$

According to the estimate (3.19), (3.31) implies that

$$
\sup _{t \in[0, T]}\left\|\nabla^{2} u\right\|_{L^{2}} \leq C \sup _{t \in[0, T]}\left\|\sqrt{\rho} \partial_{t} u\right\|_{L^{2}}+C\|\nabla u\|_{L^{2}}^{3} \leq \bar{C}
$$

It completes the proof.

Lemma 3.5 (Estimate for $\|\nabla \rho\|_{H^{1}}$ ). Under the assumptions of Theorem 3.1, we know that

$$
\sup _{t \in[0, T]}\|\nabla \rho\|_{H^{1}}+\int_{0}^{T}\left\|\nabla^{3} u\right\|_{L^{2}}^{2} d t \leq \bar{C} .
$$

Proof. Differentiating the mass equation with respect to $x_{j}, j=1,2,3$, we find that $\partial_{j} \rho$ satisfies

$$
\partial_{t} \partial_{j} \rho+u \cdot \nabla \partial_{j} \rho=-\partial_{j} u \cdot \nabla \rho .
$$

Then multiplying (3.34) by $\partial_{j} \rho$, integrating over $\mathbb{R}^{3}$, and summing over the index $j$, one gets that

$$
\frac{d}{d t} \int|\nabla \rho|^{2} d x \leq C \int|\nabla u| \cdot|\nabla \rho|^{2} d x \leq C\|\nabla u\|_{L^{\infty}}\|\nabla \rho\|_{L^{2}}^{2}
$$

To derive the appropriate bound for $\int_{0}^{T}\|\nabla u\|_{L^{\infty}} d t$, we make use of the elliptic estimates related with the momentum equation. In fact,

$$
\begin{aligned}
\left\|\nabla^{2} u\right\|_{L^{4}} & \leq C\left\|\rho \partial_{t} u\right\|_{L^{4}}+C\|(\rho u \cdot \nabla) u\|_{L^{4}} \\
& \leq C\left\|\nabla \partial_{t} u\right\|_{L^{2}}^{\frac{3}{4}}\left\|\sqrt{\rho} \partial_{t} u\right\|_{L^{2}}^{\frac{1}{4}}+C\|u\|_{L^{12}}\|\nabla u\|_{L^{6}} \\
& \leq C\left\|\nabla \partial_{t} u\right\|_{L^{2}}^{\frac{3}{4}}\left\|\sqrt{\rho} \partial_{t} u\right\|_{L^{2}}^{\frac{1}{4}}+C\|\nabla u\|_{L^{2}}^{\frac{3}{4}}\|\nabla u\|_{L^{6}}^{\frac{5}{4}}
\end{aligned}
$$


which together with Lemma 3.4 gives that

$$
\left\|\nabla^{2} u\right\|_{L^{2}\left(0, T ; L^{4}\right)} \leq \bar{C} .
$$

By virtue of the Gagliardo-Nirenberg inequality,

$$
\|\nabla u\|_{L^{\infty}} \leq C\|\nabla u\|_{L^{6}}^{\frac{1}{3}}\left\|\nabla^{2} u\right\|_{L^{4}}^{\frac{2}{3}}
$$

hence $\|\nabla u\|_{L^{1}\left(0, T ; L^{\infty}\right)} \leq \bar{C}$. Consequently, by Gronwall's inequality, (3.35) gives a bound for $\|\nabla \rho\|_{L^{2}}$. Similar argument shows that

$$
\begin{aligned}
\frac{d}{d t} \int\left|\nabla^{2} \rho\right|^{2} d x & \leq C \int\left(\left|\nabla u\left\|\left.\nabla^{2} \rho\right|^{2}+\left|\nabla^{2} u\|\nabla \rho\| \nabla^{2} \rho\right|\right) d x\right.\right. \\
& \leq C\|\nabla u\|_{L^{\infty}}\left\|\nabla^{2} \rho\right\|_{L^{2}}^{2}+C\left\|\nabla^{2} u\right\|_{L^{3}}\|\nabla \rho\|_{L^{6}}\left\|\nabla^{2} \rho\right\|_{L^{2}} \\
& \leq C\left(\|\nabla u\|_{L^{\infty}}+\left\|\nabla^{2} u\right\|_{L^{3}}\right)\left\|\nabla^{2} \rho\right\|_{L^{2}}^{2} .
\end{aligned}
$$

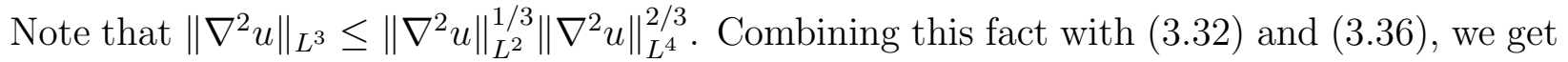
that

$$
\nabla^{2} u \in L^{1}\left(0, T ; L^{3}\right)
$$

Hence, the Gronwall inequality gives a bound for $\left\|\nabla^{2} \rho\right\|_{L^{2}}$.

Finally, using the reguality theory for Stokes equations, one can obtain that

$$
\begin{aligned}
\left\|\nabla^{3} u\right\|_{L^{2}} & \leq C\left(\left\|\rho \partial_{t} u\right\|_{H^{1}}+\|\rho u \cdot \nabla u\|_{H^{1}}\right) \\
& \leq C\left(\|\nabla \rho\|_{L^{3}}+1\right)\left(\left\|\nabla \partial_{t} u\right\|_{L^{2}}+\|\nabla u\|_{H^{1}}^{2}\right),
\end{aligned}
$$

which implies that $\left\|\nabla^{3} u\right\|_{L^{2}\left(0, T ; L^{2}\right)} \leq \bar{C}$.

3.2. Proof of Theoerem 1.2. With the a priori estimates in subsection 3.1 in hand, we are prepared for the proof of Thorem 1.2 .

Proof. According to Theorem 1.1, there exists a $T_{*}>0$ such that the Cauchy problem (1.1)-(1.2) has a unique local strong solution $(\rho, u, P)$ on $\mathbb{R}^{3} \times\left(0, T_{*}\right]$, where $T_{*}$ depends on $\left\|\rho_{0}\right\|_{L^{\frac{3}{2}} \cap H^{2}},\left\|\nabla u_{0}\right\|_{H^{1}}$ and $\|g\|_{L^{2}}$. We will show that this local solution extends to a global one.

It follows from the integrability property of the local strong solution that $A(0)=0$. Hence there exists a $T_{1} \in\left(0, T_{*}\right)$ such that (3.11) holds for $T=T_{1}$. Set

$$
T^{*}=\sup \left\{T \mid(\rho, u, P) \text { is a strong solution on } \mathbb{R}^{3} \times(0, T] \text { and } A(T) \leq 2\right\} .
$$

Then $T^{*} \geq T_{1}>0$.

The claim is that $T^{*}=\infty$, for otherwise $T^{*}<\infty$, which we will assume for an argument by contradiction. First, it follows from Lemmas 3.4 and 3.5 that

$$
\|\nabla u(t)\|_{H^{1}} \leq \bar{C}\left(T^{*}\right), \quad\|\rho(t)\|_{L^{\frac{3}{2}} \cap H^{2}} \leq \bar{C}\left(T^{*}\right), \quad \text { for every } 0<t<T^{*},
$$

where $\bar{C}\left(T^{*}\right)$ depends on $T^{*}$ and the initial data. Secondly,

$$
\mu \Delta u-\nabla P=\rho \partial_{t} u-(\rho u \cdot \nabla) u,
$$


which implies that

$$
\begin{aligned}
\left\|\rho^{-\frac{1}{2}}(\mu \Delta u-\nabla P)\right\|_{L^{2}} & \leq\left\|\sqrt{\rho} \partial_{t} u\right\|_{L^{2}}+\|(\sqrt{\rho} u \cdot \nabla) u\|_{L^{2}} \\
& \leq\left\|\sqrt{\rho} \partial_{t} u\right\|_{L^{2}}+C(\bar{\rho})\|\nabla u\|_{H^{1}}^{\frac{3}{2}} \cdot\|\sqrt{\rho} u\|_{L^{2}}^{\frac{1}{2}} \\
& \leq \bar{C}\left(T^{*}\right) .
\end{aligned}
$$

These uniform estimates allow us to construct a new unique local strong solution from $\left(\rho\left(T_{2}\right), u\left(T_{2}\right)\right)$ for any $0<T_{2}<T^{*}$, for which the life span is uniformly bounded from below by some $\Delta T$. By uniqueness, on the interval $\left[T_{2}, T^{*}\right)$, this new strong solution coincides with the original one. Choose some $T_{2}$ close enough to $T^{*}$, such that $T_{2}+\Delta T>T^{*}$, giving a local strong solution $(\rho, u, P)$ on $\left(0, T_{2}+\Delta T\right]$.

Next, we show that (3.11) holds on $\left(0, T_{2}+\Delta T\right]$. For the strong solution $(\rho, u, P)$ on $\left[0, T_{2}+\Delta T\right]$, let

$$
T^{* *}=\sup \{T \mid \quad A(T) \leq 2\} .
$$

It follows from Theorem 3.1 that in fact

$$
A\left(T^{* *}\right) \leq 1 .
$$

Hence $T^{* *}=T_{2}+\Delta T$ and (3.11) holds on $\left(0, T_{2}+\Delta T\right]$. In fact this is a contradiction to the assumption that $T^{*}<\infty$ is the maximal time, since $T_{2}+\Delta T>T^{*}$. Furthermore the decay property of the solution is implied in the proof of Lemma 3.3. Hence, the proof is complete.

\section{Proof of Theorem 1.2 for the CASE of A Bounded domain}

In this section, we assume that the domain $\Omega$ is bounded and smooth domain in $\mathbb{R}^{3}$. As before, for strong solutions $(\rho, u, P)$ to (1.1) $-(1.2)$ on $\Omega \times[0, T]$, define

$$
A(t)=\|\nabla u\|_{L^{4}\left(0, t ; L^{2}\right)}, \quad 0 \leq t \leq T .
$$

Proceeding as in the above section, we establish a priori estimates for $(\rho, u)$ which guarantee the extension of the local solution.

Theorem 4.1. Under the assumptions of Theorem 1.1, there exists some positive constant $\varepsilon$ depending only on $\mu, \Omega$ and $\bar{\rho}$, such that if $\left\|u_{0}\right\|_{\dot{H}^{\frac{1}{2}}} \leq \varepsilon$ and $(\rho, u, P)$ is a strong solution to (1.1) -(1.2), satisfying

$$
A(T) \leq 2
$$

then it in fact holds that

$$
A(T) \leq 1 .
$$

The proof for Theorem 4.1 is similar to that for Theorem 3.1, with some slight changes due to the presence of several additional lower order terms that appear because of the existence of a boundary. These terms are controlled using the exponential decay of the kinetic energy.

Lemma 4.2. Let $(\rho, u, P)$ be a strong solution to (1.1)-(1.2). Then for every $0 \leq t \leq T$,

$$
\|\rho(t)\|_{L^{\infty}}=\left\|\rho_{0}\right\|_{L^{\infty}}=\bar{\rho} \text {. }
$$


Lemma 4.3. [Modified Energy Estimate] Let $(\rho, u, P)$ be a strong solution to (1.1)-(1.2). Then it satisfies

$$
\|\sqrt{\rho} u(t)\|_{L^{2}} \leq\left\|\sqrt{\rho_{0}} u_{0}\right\|_{L^{2}} \exp \{-C(\mu, \Omega, \bar{\rho}) t\} .
$$

Proof. According to the basic energy estimate,

$$
\frac{1}{2} \frac{d}{d t} \int \rho|u|^{2} d x+\mu \int|\nabla u|^{2} d x=0 .
$$

By the Poincaré inequality,

$$
\int \rho|u|^{2} d x \leq C(\Omega, \bar{\rho})\|\nabla u\|_{L^{2}}^{2},
$$

which, when incorporated into (4.43), yields

$$
\frac{d}{d t} \int \rho|u|^{2} d x+\frac{2 \mu}{C(\Omega, \bar{\rho})} \int \rho|u|^{2} d x \leq 0 \text {. }
$$

Consequently

$$
\int \rho(t)|u(t)|^{2} d x \leq \int \rho_{0}\left|u_{0}\right|^{2} d x \cdot \exp \{-C(\mu, \Omega, \bar{\rho}) t\}
$$

Lemma 4.4. Let $(\rho, u, P)$ be a strong solution to (1.1) -(1.2). Then

$$
\sup _{t \in[0, T]} t \int \rho|u|^{2} d x+\int_{0}^{T} t \int|\nabla u|^{2} d x d t \leq C \int \rho_{0}\left|u_{0}\right|^{2} d x .
$$

Proof. Multiplying the momentum equation by $t u$ and integrating over $\Omega$,

$$
\frac{t}{2} \frac{d}{d t} \int \rho|u|^{2} d x+\mu t \int|\nabla u|^{2} d x=0
$$

which implies that

$$
\sup _{t \in[0, T]} \frac{t}{2} \int \rho|u|^{2} d x+\mu \int_{0}^{T} t \int|\nabla u|^{2} d x d t=\frac{1}{2} \int_{0}^{T} \int \rho|u|^{2} d x d t .
$$

Taking (4.42) into account, one finds that

$$
\sup _{t \in[0, T]} t \int \rho|u|^{2} d x+\mu \int_{0}^{T} t \int|\nabla u|^{2} d x d t \leq C \int \rho_{0}\left|u_{0}\right|^{2} d x,
$$

which completes the proof.

Lemma 4.5. Let $(\rho, u, P)$ be a strong solution of the equations (1.1)-(1.2). Suppose that

$$
A(T) \leq 2,
$$

then there exists some constant $C_{1}$ depending on $\mu, \bar{\rho}, \Omega$, such that

$$
\|\nabla u\|_{L^{4}\left(0, T ; L^{2}\right)} \leq C_{1}\left\|u_{0}\right\|_{\dot{H}^{\frac{1}{2}}},
$$


and it holds that

$$
\sup _{t \in[0, T]} t\|\nabla u(t)\|_{L^{2}}^{2} \leq C \int \rho_{0}\left|u_{0}\right|^{2} d x .
$$

Note that $C_{1}$ does not depend on $T$.

Proof. The proof is similar as in the case of the whole space. We consider the following linear system for $(w, \tilde{P})$,

$$
\left\{\begin{array}{l}
\rho \partial_{t} w-\mu \Delta w+(\rho u \cdot \nabla) w+\nabla \tilde{P}=0, \quad \text { in } \Omega \times(0, T], \\
\operatorname{div} w=0, \quad \text { in } \Omega \times[0, T], \\
w=0, \quad \text { on } \partial \Omega \times[0, T], \\
w(x, 0)=w_{0}(x), \quad \text { in } \Omega .
\end{array}\right.
$$

Suppose $w_{0}$ satisfies the conditions assumed of $u_{0}$ in Theorem 1.1, then the global existence and uniqueness of strong solution to (4.48) is known. Straightforward energy estimates tell that

$$
\frac{1}{2} \int \rho|w(T)|^{2} d x+\mu \int_{0}^{T}\|\nabla w\|_{L^{2}}^{2} d t \leq \frac{1}{2} \int \rho_{0}\left|w_{0}\right|^{2} d x \leq C(\bar{\rho})\left\|w_{0}\right\|_{L^{2}}^{2} .
$$

Multiplying (4.48) $)_{1}$ by $\partial_{t} w$ and integrating over $\Omega$, one gets by the Sobolev embedding theorem that

$$
\begin{array}{rl}
\int \rho\left|\partial_{t} w\right|^{2} & d x+\frac{\mu}{2} \frac{d}{d t} \int|\nabla w|^{2} d x=-\int \rho u \cdot \nabla w \cdot \partial_{t} w d x \\
& \leq C\|u\|_{L^{6}}\|\nabla w\|_{L^{3}}\left\|\sqrt{\rho} \partial_{t} w\right\|_{L^{2}} \\
& \leq \frac{1}{4} \int \rho\left|\partial_{t} w\right|^{2} d x+C\|\nabla u\|_{L^{2}}^{2}\|\nabla w\|_{L^{2}}\|\nabla w\|_{H^{1}}
\end{array}
$$

From the regularity theory for stationary Stokes system, it follows that

$$
\begin{aligned}
& \|\nabla w\|_{H^{1}} \leq C\left(\left\|\rho \partial_{t} w\right\|_{L^{2}}+\|(\rho u \cdot \nabla) w\|_{L^{2}}\right)+C\|\nabla w\|_{L^{2}} \\
& \quad \leq C\left\|\rho \partial_{t} w\right\|_{L^{2}}+C\|\nabla u\|_{L^{2}}\|\nabla w\|_{L^{2}}^{\frac{1}{2}}\|\nabla w\|_{H^{1}}^{\frac{1}{2}}+C\|\nabla w\|_{L^{2}},
\end{aligned}
$$

which implies

$$
\|\nabla w\|_{H^{1}} \leq C\left\|\rho \partial_{t} w\right\|_{L^{2}}+C\|\nabla u\|_{L^{2}}^{2}\|\nabla w\|_{L^{2}}+C\|\nabla w\|_{L^{2}} .
$$

Inserting the estimate (4.51) in (4.50), then

$$
\begin{aligned}
& \int \rho\left|\partial_{t} w\right|^{2} d x+\mu \frac{d}{d t} \int|\nabla w|^{2} d x \\
& \leq C\|\nabla u\|_{L^{2}}^{4}\|\nabla w\|_{L^{2}}^{2}+C\|\nabla u\|_{L^{2}}^{2}\|\nabla w\|_{L^{2}}^{2} \\
& \leq C\|\nabla u\|_{L^{2}}^{4}\|\nabla w\|_{L^{2}}^{2}+\mu\|\nabla w\|_{L^{2}}^{2} .
\end{aligned}
$$


Utilizing Gronwall's inequality and Poincaré inequality,

$$
\begin{gathered}
\int_{0}^{T}\left\|\sqrt{\rho} \partial_{t} w\right\|_{L^{2}}^{2} d t+\mu\|\nabla w(T)\|_{L^{2}}^{2} \leq C e^{C}\left\|\nabla w_{0}\right\|_{L^{2}}^{2}+C \mu \int_{0}^{T}\|\nabla w\|_{L^{2}}^{2} d t \\
\leq C e^{C}\left\|\nabla w_{0}\right\|_{L^{2}}^{2}+C \int \rho_{0}\left|w_{0}\right|^{2} d x \leq C e^{C}\left\|\nabla w_{0}\right\|_{L^{2}}^{2} .
\end{gathered}
$$
that

By interpolation, $L^{4}\left(0, T ; L^{2}\right)=\left[L^{2}\left(0, T ; L^{2}\right), L^{\infty}\left(0, T ; L^{2}\right)\right]_{\frac{1}{2}, 4}$ and $\dot{H}^{\frac{1}{2}} \subseteq\left[L^{2}, \dot{H}^{1}\right]_{\frac{1}{2}, 4}$, so

$$
\|\nabla w\|_{L^{4}\left(0, T ; L^{2}\right)} \leq C_{1}\left\|w_{0}\right\|_{\dot{H}^{\frac{1}{2}}}
$$

which also holds for $u$.

Furthermore, multiplying (4.52) by $t$ we have

$$
t \int \rho\left|\partial_{t} u\right|^{2} d x+\mu \frac{d}{d t} \int t|\nabla u|^{2} d x \leq \mu \int|\nabla u|^{2} d x+C t\|\nabla u\|_{L^{2}}^{6}+\mu t\|\nabla u\|_{L^{2}}^{2} .
$$

By Gronwall's inequality and Lemma 4.4, for every $t \in[0, T]$,

$$
\int_{0}^{t} s \int \rho\left|\partial_{t} u(s)\right|^{2} d x d s+t\|\nabla u\|_{L^{2}}^{2} \leq C \int_{0}^{t}(1+s)\|\nabla u\|_{L^{2}}^{2} d s \leq C \int \rho_{0}\left|u_{0}\right|^{2} d x
$$

which completes the proof.

The proof of Theorem 4.1 is thus complete, if we set $\varepsilon \leq 1 / C_{1}$. Finally, the proof of Theorem 1.2 for bounded domain case follows the same logic as the analog result in section 3 , we omit the details for reasons of brevity.

\section{REFERENCES}

[1] H. Abidi and M. Paicu, Existence globale pour un fluide inhomogéne, Ann. Inst. Fourier (Grenoble) 57 (2007), 883-917.

[2] Adams, Robert A, Sobolev spaces, Academic Press, New York-London, 1975.

[3] S. Agmon, A. Douglis and L. Nirenberg, Estimates near the boundary for solutions of elliptic partial differential equations satisfying general boundary conditions, Comm. Pure Appl. Math., 12 (1959), No. 4, 623-727.

[4] S. A. Antontesv, A. V. Kazhikov and V. N. Monakhov, Boundary Value Problems in Mechanics of Nonhomogeneous Fluids, North-Holland, Amsterdam, 1990.

[5] H. Bahouri, J. Y. Chemin and R. Danchin, Fourier Analysis and Nonlinear Partial Differential Equations, Springer, 2011.

[6] H. Brezis and T. Gallouet, Nonlinear Schrödinger evolution equations, Nonlinear Anal. T. M. A. , 4 (1980), 677-681.

[7] H. Brezis and S. Wainger, A note on limiting cases of Sobolev embedding and convolution inequalities, Comm. Partial Differential Equations, 5 (1980), 773-789.

[8] J. Bergh and J. Löfström, Interpolation spaces. An introduction. Grundlehren der Mathematischen Wissenschaften, No. 223. Springer-Verlag, Berlin-New York, 1976.

[9] M. Cannone, Harmonic analysis tools for solving the incompressible NavierStokes equations, in: Handbook of Mathematical Fluid Dynamics, vol. III, North-Holland, Amsterdam, 2004, pp. 161-244.

[10] J.-Y. Chemin, I. Gallagher and M. Paicu, Global regularity for some classes of large solutions to the Navier -Stokes equations. Ann. of Math.173 (2011), no. 2, pp. 983-1012. 
[11] Y. Cho and H. Kim, Unique solvability for the density-dependent Navier-Stokes equations, Nonlinear Anal. 59 (2004), no. 4, 465-489.

[12] H. Y. Choe and H. Kim, Strong solutions of the NavierStokes equations for nonhomogeneous incompressible fluids, Comm. Partial Differential Equations, 28 (2003) no. 5-6, 1183-1201.

[13] R. Danchin, Density-dependent incompressible viscous fluids in critical spaces, Proc. Roy. Soc. Edinburgh Sect. A, 133 (2003), 1311-1334.

[14] B. Desjardins, Regularity results for two-dimensional flows of multiphase viscous fluids, Arch. Ration. Mech. Anal., 137 (1997), 135-158.

[15] R. J. DiPerna and P. L. Lions, Equations différentielles ordinaires et équations de transport avec des coefficients irréguliers, in: Séminaire EDP 1988-1989, Ecole Polytechnique, Palaiseau, 1989.

[16] H. Fujita and T. Kato, On the NavierStokes initial value problem. I, Arch. Ration. Mech. Anal. 16 (1964), 269-315.

[17] G. P. Galdi, An introduction to the Mathematical Theory of Navier-Stokes Equations, Vol. I: Linearized Steady Problems, Springer Verlag, 381994.

[18] G. L. Gui, J. C. Huang and P. Zhang, Large global solutions to 3-D inhomogeneous Navier-Stokes equations slowly varying in one variable, J. Funct. Analysis, 261 (2011), 3181-3210.

[19] X. D. Huang, J. Li and Z. P. Xin, Global well-posedness of classical solutions with large oscillations and vacuum to the three-dimensional isentropic compressible Navier-Stokes equations, Comm. Pure Appl. Math. 65(4), 549-585(2012).

[20] X. D. Huang and Y. Wang, Global strong solution with vacuum to the 2D nonhomogeneous incompressible MHD system, http://arxiv.org/abs/1206.6144.

[21] A. V. Kazhikov, Resolution of boundary value problems for nonhomogeneous viscous fluids, Dokl. Akad. Nauk., 216 (1974), 1008-1010.

[22] H. Kim, A blow-up criterion for the nonhomogeneous incompressible Navier-Stokes equations, SIAM J. Math. Anal., 37 (2006), no. 5, 1417-1434.

[23] H. Koch and D. Tataru, Well-posedness for the Navier - Stokes equations, Adv. Math. 15 (2001), no. $1,22-35$.

[24] O. Ladyzhenskaya and V. A. Solonnikov, Unique solvability of an initial and boundary value problem for viscous incompressible non-homogeneous fluids, J. Soviet Math., 9 (1978), 697-749.

[25] P. L. Lions, Mathematical Topics in Fluid Mechanics, Vol. I: Incompressible Models, Oxford Lecture Series in Math. and Its Appl., 3, Oxford University Press, New York, 1996.

[26] J. Serrin, The initial value problem for the Navier-Stokes equations, In Nonlinear Problems, University of Wisconsin Press, Madison, Wis., 1963, pp. 69-98.

[27] J. Simon, Nonhomogeneous viscous incompressible fluids: Existence of velocity, density, and pressure, SIAM J. Math. Anal. , 21 (1990), 1093-1117.

[28] L. Tartar, An Introduction to Sobolev Spaces and Interpolation Spaces, Springer, 2007.

[29] H. Tribel, Interpolation theory, Function Spaces, Differential Operators, North-Holland Mathematical Theory, 18, North-Holland Publishing Co., Amsterdam-New York, 1978.

Department of Mathematics and Statistics, McMaster University, 1280 Main Street

West, Hamilton, Ontario L8S 4K1, Canada

E-mail address: craig@math.mcmaster.ca

Academy of Mathematics and Systems Science, CAS, Beijing 100190, P. R. China \& Department of Pure and Applied Mathematics, Graduate School of Information Sciences and Technology, Osaka University, Osaka, Japan

E-mail address: xdhuang@amss.ac.cn

Department of Mathematics and Statistics, McMaster University, 1280 Main Street West, Hamilton, Ontario L8S 4K1, Canada

E-mail address: yunwang@math.mcmaster.ca 